\title{
Efecto del volumen radicular y la tasa de fertilización sobre el comportamiento en terreno de plantas de pino oregón (Pseudotsuga menziesii (Mirb.) Franco) producidas con el método $1+1$
}

\author{
Effects of preplanting root volume and fertilization rate on field performance of 1+1 Douglas-fir \\ (Pseudotsuga menziesii (Mirb.) Franco) seedlings \\ PATRICIO ALZUGARAY', DIANE HAASE², ROBIN ROSE ${ }^{2}$ \\ ${ }^{1}$ Instituto Forestal Sede Bío-Bío, camino a Coronel km 7.5, Concepción-Chile. e-mail: palzugar@infor.cl. \\ ${ }^{2}$ Nursery Technology Cooperative, Oregon State University, Corvallis, OR, 97331-5752, Estados Unidos. \\ E-mail:diane.haase@oregonstate.edu.; robin.rose@oregonstate.edu.
}

\begin{abstract}
SUMMARY
To determine the combined effects of the preplanting root volume and fertilization rate on seedling field performance, Douglas-fir $1+1$ seedlings, grown using standard practices at the Weyerhaeuser Aurora nursery (Oregon, USA), were sorted into four root-volume categories $\left(8-13,14-17,18-22\right.$, and $\left.23-35 \mathrm{~cm}^{3}\right)$, and planted in a recent clearcut in Oregon State University's McDonald-Dunn Research Forest on January 19, 2000 using a complete randomized block design with five replicates in a factorial treatment arrangement. A Simplot Polyon ${ }^{\circledR}$ fertilizer mixture, containing equal parts of three fertilizers with different release rates (3-4, 5-6, and 8-9 months) and similar NPK concentrations (19-6-12) was applied at the time of planting in the bottom of the planting hole to each root-volume category at five rates: 0 (unfertilized control), 15, 30, 45 and $60 \mathrm{~g}$ per seedling. After two growing seasons in the field, there was no interaction between root volume and fertilization rate for seedling growth and survival. Seedlings with a larger preplanting root volume had greater survival and growth than seedlings with a smaller root volume. There was a significant reduction in seedling survival and growth with increasing fertilization rate.
\end{abstract}

Key words: reforestation, seedling quality, root-volume, fertilization, Douglas-fir.

\section{RESUMEN}

Con el fin de determinar los efectos combinados del volumen radicular y la tasa de fertilización sobre el comportamiento en terreno de plantas de pino oregón producidas a través del método $1+1$, plantas producidas operacionalmente en el vivero de la empresa Weyerhaeuser ubicado en Aurora, OR, Estados Unidos, fueron clasificadas en cuatro categorías de acuerdo a su volumen radicular $\left(8-13,14-17,18-22\right.$, y $\left.23-35 \mathrm{~cm}^{3}\right)$, y establecidas en terreno durante el invierno del 2000, utilizando un diseño de bloques aleatorios con cinco réplicas en un arreglo factorial. Una mezcla de fertilizantes de entrega controlada (Simplot) que contenía iguales proporciones de tres fertilizantes con diferentes efectos residuales (3-4, 5-6, y 8-9 meses) y similares concentraciones de NPK (19-6-12), fue aplicada en el fondo del hoyo de plantación en cinco dosis: 0, 15, 30, 45 y 60 g por planta. Después de dos temporadas de crecimiento en terreno, no existió interacción entre el volumen radicular y la dosis de fertilización para la supervivencia y crecimiento inicial de las plantas. Aquellas plantas con mayor volumen radicular sobrevivieron y se desarrollaron mejor que aquellas con menor volumen radicular. El aumento de la tasa de fertilización redujo significativamente la supervivencia y el crecimiento de las plantas.

Palabras clave: reforestación, calidad de plantas, volumen radicular, fertilización, pino oregón. 
BOSQUE 25(2): 17-33, 2004

Efecto del volumen radicular y la tasa de fertilización sobre el comportamiento en terreno...

\section{INTRODUCCION}

Durante las primeras etapas de una plantación forestal, el desarrollo de las plantas está condicionado a los recursos disponibles del sitio en el que fueron establecidas. El uso de plantas de buena calidad, capaces de sobrevivir y crecer vigorosamente una vez plantadas junto con la aplicación de tratamientos silviculturales de preparación de suelos, control de vegetación competitiva y fertilización contribuyen a reducir el tiempo requerido por la especie introducida para la ocupación completa del sitio (1).

La calidad de las plantas está determinada por su comportamiento en terreno. Plantas de buena calidad son aquellas capaces de sobrevivir estreses ambientales prolongados y crecer vigorosamente inmediatamente después de plantadas en un sitio particular (2). Bajo una óptima condición fisiológica, la morfología de las plantas es un buen indicador de su calidad (3). Entre los parámetros morfológicos, los más usados por viveristas para clasificar las plantas por calidad han sido la longitud y diámetro del tallo $(4,5)$. Sin embargo, no siempre han sido confiables, especialmente cuando plantas excesivamente altas son establecidas en sitios de escasa disponibilidad de agua (6).

Varios autores han sugerido incluir el tamaño del sistema radicular de las plantas como un criterio para estimar su calidad (7-10). El volumen radicular de las plantas es un atractivo criterio para estimar la calidad de las plantas y predecir su comportamiento en terreno una vez plantadas, ya que puede ser medido en plantas producidas a raíz desnuda y raíz cubierta a través de métodos no destructivos $(11,12)$. Si es medido justo antes de la plantación, el volumen radicular ha sido directamente correlacionado con la supervivencia y crecimiento inicial de las plantas en terreno (8-10). Plantas con mayores volúmenes radiculares son capaces de superar más fácilmente el shock de trasplante (13), presentan un mayor potencial de crecimiento radicular (7), capacidad de absorción de agua (7) y nutrientes (14). El volumen radicular de las plantas está positivamente correlacionado con la longitud y diámetro del tallo, y la biomasa total de las plantas $(8,9,13)$. Además, las diferencias iniciales en tamaño de las plantas se mantienen con el transcurso del tiempo (10).

Por otro lado, la fertilización aplicada al momento de la plantación se ha convertido en una práctica común utilizada mundialmente con el fin de mejorar el estatus nutricional del suelo a través de la adición antropogénica de elementos minerales y con el fin de promover el crecimiento inicial de las plantas (15-17). Tradicionalmente se han aplicado fertilizantes solubles como superfosfato triple y urea; sin embargo, con el advenimiento de la tecnología de fertilizantes de entrega controlada, la aplicación de estos productos está ganando creciente interés en el mundo. La fertilización con productos de entrega controlada permite prolongar el período a través del cual dosis adecuadas de nutrientes son entregadas a las plantas, minimizando el riesgo de toxicidad por sobredosis.

Este estudio fue diseñado con el fin de determinar el efecto combinado del volumen radicular y la dosis de fertilización sobre la supervivencia y crecimiento inicial de plantas de pino oregón producidas a raíz desnuda a través del método $1+1$.

\section{MATERIAL Y METODOS}

Una muestra aleatoria de 2.570 plantas producidas operacionalmente a raíz desnuda a través del método $1+1$ en el vivero de la empresa Weyerhaeuser en Aurora, OR, Estados Unidos, fue tomada y transportada al laboratorio de la Cooperativa Tecnológica de Viveros (NTC) en la Universidad de Oregon State, donde fueron lavadas y clasificadas de acuerdo a la distribución de su volumen radicular (figura 1), medido por desplazamiento de agua (11) en cuatro categorías: RV1 $\left(8-13 \mathrm{~cm}^{3}\right), \operatorname{RV} 2\left(14-17 \mathrm{~cm}^{3}\right), \operatorname{RV} 3\left(18-22 \mathrm{~cm}^{3}\right), y$ RV4 (23-35 $\left.\mathrm{cm}^{3}\right)$. Los rangos de las diferentes categorías fueron determinados basados en su distribución con el fin de obtener 500 plantas por categoría.

Los tratamientos de fertilización consistieron en la aplicación de un fertilizante de entrega controlada al momento de la plantación, en cinco dosis diferentes: 0 (testigo), 15, 30, 45 y 60 g por planta. El fertilizante fue aplicado en el fondo del hoyo de plantación cubierto con una delgada capa de suelo y enseguida la planta se plantó sobre el fertilizante. El fertilizante utilizado en este estudio fue fabricado por la empresa Simplot y contenía iguales proporciones de tres diferentes fertilizantes de entrega controlada con diferentes efectos residuales (cuadro 1). 
BOSQUE 25(2): 17-33, 2004 Efecto del volumen radicular y la tasa de fertilización sobre el comportamiento en terreno...

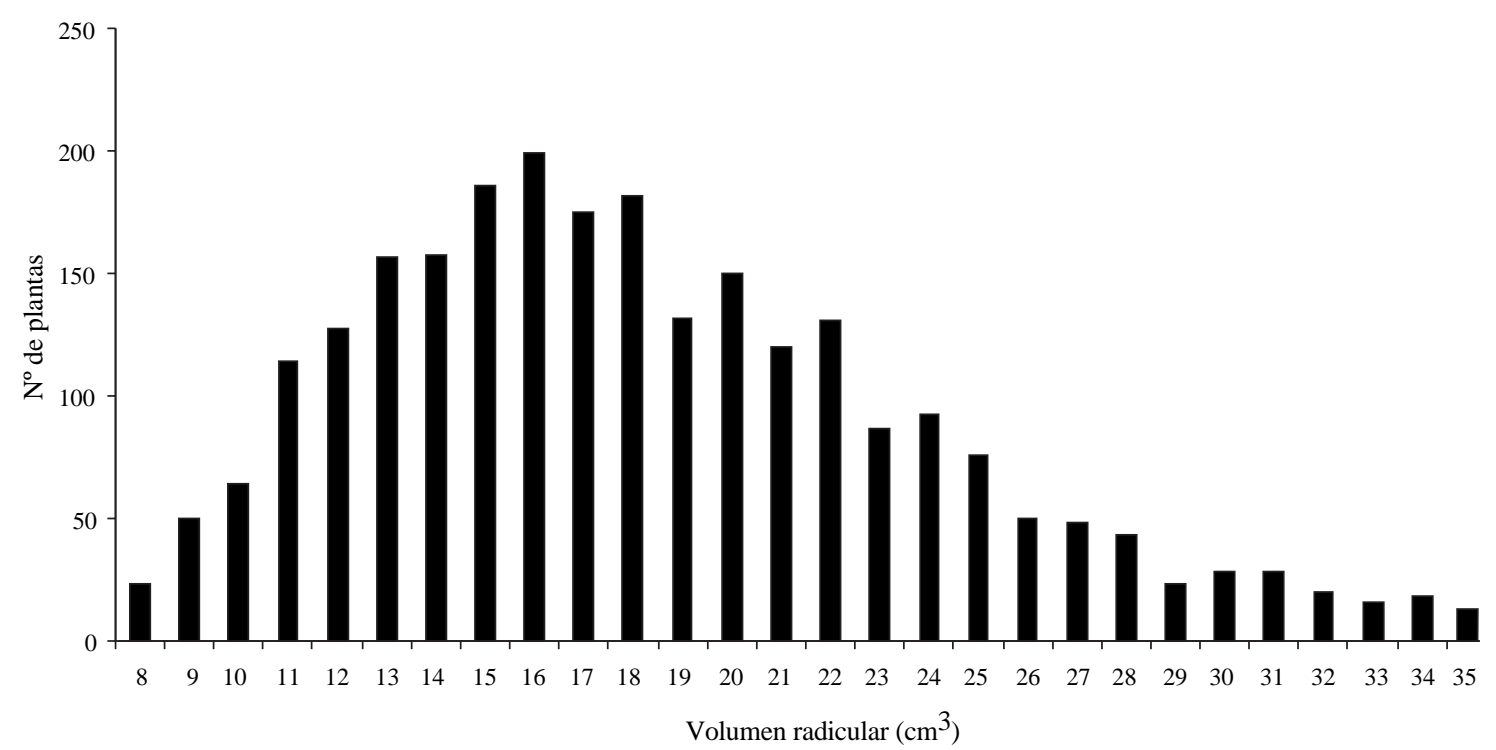

Figura 1. Distribución del volumen radicular de las 2.000 plantas utilizadas en el estudio. Root-volume distribution for the 2.000 seedlings used in the experiment.

CUADRO 1

Composición nutricional (\%) de los tres fertilizantes Simplot Polyon utilizados para originar la mezcla utilizada en este estudio.

Nutrient composition (\%) of the three Simplot Polyon fertilizers used to create the composite fertilizer applied in this experiment.

\begin{tabular}{|l|c|c|c|c|}
\hline $\begin{array}{c}\text { Fertilizante } \\
\text { Efecto residual }\end{array}$ & $\begin{array}{c}\text { Polyon 1 } \\
(3-4 \text { meses })\end{array}$ & $\begin{array}{c}\text { Polyon 2 } \\
(5-6 \text { meses })\end{array}$ & $\begin{array}{c}\text { Polyon 3 } \\
(8-9 \text { meses })\end{array}$ & $\begin{array}{c}\text { Mezcla } \\
\text { aplicada }\end{array}$ \\
\hline Nutriente & \multicolumn{4}{|c|}{ Composición (\%) } \\
\hline Nitrógeno total & 19 & 19 & 18 & 18,67 \\
$\mathrm{NH}_{4}$ & 8,2 & 8,2 & 7,74 & 8,05 \\
$\mathrm{NO}_{3}$ & 10,8 & 10,8 & 10,26 & 10,62 \\
$\mathrm{P}_{2} \mathrm{O}_{5}$ & 5 & 6 & 6 & 5,67 \\
$\mathrm{~K}_{2} \mathrm{O}$ & 12 & 12 & 12 & 12 \\
$\mathrm{Mg}$ & 1 & 0,9 & 0,9 & 0,93 \\
$\mathrm{~S}$ & 1,8 & 1,7 & 1,7 & 1,73 \\
$\mathrm{Fe}$ & 0,45 & 0,45 & 0,45 & 0,45 \\
$\mathrm{Mn}$ & 0,2 & 0,19 & 0,19 & 0,193 \\
$\mathrm{Mo}$ & 0,009 & 0,009 & 0,009 & 0,009 \\
$\mathrm{Zn}$ & 0,056 & 0,055 & 0,05 & 0,054 \\
\hline
\end{tabular}


BOSQUE 25(2): 17-33, 2004

Efecto del volumen radicular y la tasa de fertilización sobre el comportamiento en terreno...

Instalación del estudio: El estudio fue establecido en enero del 2000, utilizando un espaciamiento de 3 x 3 metros en un sitio cosechado durante el año 1998 en un predio de la Universidad de Oregon State, ubicado $15 \mathrm{~km}$ al norte de la ciudad de Corvallis, OR, aproximadamente. La vegetación competitiva fue controlada antes y después del establecimiento del estudio con aplicaciones de Accord $^{\circledR}$, Arsenal $^{\circledR}$, Oust $^{\circledR}$ y Escort ${ }^{\circledR}$ (preplan- $^{-}$ tación), y Transline ${ }^{\circledR}$, Garlon $4^{\circledR}$ y Atrazine ${ }^{\circledR}$ (postplantación). El sitio tiene una exposición Este y los suelos son limo-arcillosos profundos de buen drenaje. El cuadro 2 muestra las características químicas de los suelos. Las precipitaciones mensuales en la ciudad de Corvallis, OR, se muestran en el cuadro 3, como una estimación de lo que podría haber precipitado en el sitio experimental. Además, un termómetro de suelos conectado a un capturador de datos fue instalado en el sitio de plantación a una profundidad de $30 \mathrm{~cm}$ para registrar temperatura de suelo mínima, promedio y máxima cada tres días.

Diseño experimental: Las plantas fueron establecidas en terreno utilizando un diseño de bloques aleatorios con cinco réplicas en un arreglo de tratamientos factorial $4 \times 5$ (cuatro categorías de volumen radicular x 5 dosis de fertilización). Cada tratamiento fue asignado aleatoriamente a una hilera de plantación de 20 plantas. Cada bloque consistió de 20 hileras de 20 plantas cada una, totalizando 2.000 plantas.
Toma de datos: Además de las 2.000 plantas utilizadas en el establecimiento del estudio, 25 plantas de cada categoría de volumen radicular fueron seleccionadas al azar para efectuar mediciones de longitud, diámetro y volumen del tallo, y volumen radicular. A continuación las plantas fueron secadas por 48 horas a $68^{\circ} \mathrm{C}$ para determinar biomasa de cada uno de sus componentes. El follaje de cinco plantas fue molido y combinado para obtener tres muestras para determinación de concentración y contenido nutricional por categoría. El contenido nutricional fue estimado a través de la multiplicación de la concentración nutricional por el peso seco de 100 acículas.

Supervivencia, altura y diámetro de las plantas se midieron en terreno dentro de un mes después de plantadas (febrero del 2000) y durante julio del 2000, septiembre del 2000 y septiembre del 2001. Al término de la primera temporada de crecimiento, tres plantas por tratamiento por bloque fueron seleccionadas aleatoriamente, excavadas y llevadas al laboratorio para un análisis morfológico. La altura total, crecimiento en altura, diámetro de tallo y diámetro de la yema terminal, volumen de tallo y volumen radicular fue medido en cada planta. El diámetro de la yema terminal fue medido en la sección más ancha de la yema.

Análisis estadístico: Análisis de correlación fue utilizado para estudiar la relación entre el volu-

\section{CUADRO 2}

Características químicas del suelo en el sitio experimental.

Soil chemical characteristics at the study site.

\begin{tabular}{|c|c|c|c|c|c|c|c|c|}
\hline $\begin{array}{c}\text { Materia } \\
\text { orgánica }\end{array}$ & $\mathrm{NO}_{3}$ & $\mathrm{P}$ & $\mathrm{K}$ & $\mathrm{Mg}$ & $\mathrm{Ca}$ & $\mathrm{Na}$ & $\mathrm{SO}_{4}$ & $\mathrm{Zn}$ \\
\hline \multirow{2}{*}{$(\%)$} & $(\mathrm{ppm})$ & $(\mathrm{ppm})$ & $(\mathrm{ppm})$ & $(\mathrm{ppm})$ & $(\mathrm{ppm})$ & $(\mathrm{ppm})$ & $(\mathrm{ppm})$ & $(\mathrm{ppm})$ \\
12,3 & 25,8 & 5,5 & 217,5 & 182,3 & $1.034,5$ & 11,3 & 3,3 & 0,2 \\
\hline & $\mathrm{Mn}$ & $\mathrm{Fe}$ & $\mathrm{Cu}$ & $\mathrm{B}$ & $\mathrm{CIC}$ & $\mathrm{CE}$ & $\mathrm{pH}$ & \\
\cline { 2 - 7 } & $(\mathrm{ppm})$ & $(\mathrm{ppm})$ & $(\mathrm{ppm})$ & $(\mathrm{ppm})$ & $($ meq/100g) & $(\mathrm{dS} / \mathrm{m})$ & & \\
& 5,5 & 16,5 & 2,5 & 0,2 & 9,4 & 0,4 & 5,7 & \\
\cline { 2 - 7 } & & &
\end{tabular}




\section{CUADRO 3}

Temperaturas de suelo promedio mensual en el sitio experimental y precipitación mensual en Corvallis, OR.

Monthly average soil temperatures at the study site and monthly rainfall at Corvallis, OR.

\begin{tabular}{|l|c|c|c|}
\hline Fecha & $\begin{array}{c}\text { Temperatura de suelo } \\
\text { promedio }{ }^{\circ} \mathrm{C}\end{array}$ & $\begin{array}{c}\text { Temperatura de suelo } \\
\text { máxima }{ }^{\circ} \mathrm{C}\end{array}$ & $\begin{array}{c}\text { Precipitación mensual } \\
(\mathrm{mm})\end{array}$ \\
\hline Feb-00 & - & - & 151 \\
Mar-00 & 7,6 & 10,9 & 88 \\
Abr-00 & 11,4 & 24,4 & 41 \\
May-00 & 13,7 & 18,5 & 70 \\
Jun-00 & 17,6 & 18,9 & 30 \\
Jul-00 & 19,5 & 20,6 & 4 \\
Ago-00 & 20,1 & 21,1 & 0 \\
Sep-00 & 17,2 & 18,2 & 14 \\
Oct-00 & 13,1 & 13,7 & 75 \\
Nov-00 & 7,8 & 9,0 & 70 \\
Dic-00 & 6,0 & 9,2 & 108 \\
Ener-01 & 5,5 & 6,2 & 36 \\
Febr-01 & 5,7 & 6,2 & 28 \\
Marz-01 & 7,9 & 8,5 & 71 \\
Abr-01 & 9,4 & 10,3 & 55 \\
\hline
\end{tabular}

men radicular y las demás variables morfológicas. Análisis de varianza de una vía fue utilizado para determinar diferencias en las variables morfológicas entre categorías de volumen radicular. Análisis de varianza de dos vías fue usado para determinar los efectos del volumen radicular y la dosis de fertilización sobre la supervivencia, los incrementos en altura y diámetro medidos en terreno y las variables morfológicas de las plantas excavadas de terreno. Análisis de covarianza fue realizado para remover las fuentes de variación debido a la altura y diámetro inicial en la determinación de los efectos de los factores sobre el crecimiento final en altura y diámetro de las plantas medidos en terreno, respectivamente. El test de Tuckey fue usado para identificar diferencias significativas entre medias de tratamientos.

\section{RESULTADOS}

Morfología y estatus nutricional preplantación: El volumen radicular estuvo positivamente correlacionado con las variables morfológicas diá- metro de tallo $(\mathrm{r}=0,81 ; \mathrm{p}<0,0001)$, longitud del tallo $(\mathrm{r}=0,54 ; \mathrm{p}<0,0001)$, volumen del tallo $(\mathrm{r}=0,82$; $\mathrm{p}<0,0001)$, biomasa foliar $(\mathrm{r}=0,81 ; \mathrm{p}<0,0001)$, biomasa del tallo $(\mathrm{r}=0,78 ; \mathrm{p}<0,0001)$, biomasa aérea $(\mathrm{r}=0,82 ; \mathrm{p}<0,0001)$ y radicular $(\mathrm{r}=0,93$; $\mathrm{p}<0,0001)$. Esto implica que en la medida en que el volumen radicular aumentó así también aumentaron los parámetros morfológicos evaluados. La relación tallo-raíz no fue afectada por el volumen radicular $(\mathrm{p}=0,65)$. Para las cuatro categorías esta variable fue levemente superior a 1 , lo que significa que las plantas mantuvieron un balance en su relación de absorción y transpiración de agua a pesar de presentar sistemas radiculares diferentes en tamaño (cuadro 4).

Tanto la concentración foliar de nutrientes como el contenido foliar de nutrientes no difirieron significativamente entre las diferentes categorías de volumen radicular (cuadros 5 y 6 ).

Comportamiento en terreno: La interacción entre la categoría de volumen radicular y la dosis de fertilización no fue estadísticamente significativa para las variables altura total, diámetro de 
BOSQUE 25(2): 17-33, 2004

Efecto del volumen radicular y la tasa de fertilización sobre el comportamiento en terreno...

\section{CUADRO 4}

Características morfológicas preplantación por categoría de volumen radicular. Preplanting morphological characteristics by root-volume category.

\begin{tabular}{|c|c|c|c|c|}
\hline Variable & Promedio & Desviación estándar & Coef. de variación (\%) & Rango \\
\hline \multicolumn{5}{|c|}{ Volumen radicular $\left(\mathrm{cm}^{3}\right)$} \\
\hline RV1 & $11,4 \mathrm{a}$ & 1,44 & 12,63 & $8,00-13,00$ \\
\hline RV2 & $15,68 \mathrm{~b}$ & 1,14 & 7,27 & $14,00-17,00$ \\
\hline RV3 & $19,80 \mathrm{c}$ & 1,50 & 7,58 & $18,00-22,00$ \\
\hline RV4 & $26,48 \mathrm{~d}$ & 3,68 & 13,90 & $23,00-35,00$ \\
\hline \multicolumn{5}{|c|}{ Longitud de tallo (cm) } \\
\hline RV1 & 26,96 a & 4,32 & 16,02 & $18,00-41,50$ \\
\hline RV2 & $31,12 \mathrm{~b}$ & 5,29 & 17,00 & $22,50-41,00$ \\
\hline RV3 & $32,04 \mathrm{~b}$ & 5,07 & 15,82 & $21,50-44,00$ \\
\hline RV4 & $35,68 \mathrm{c}$ & 5,52 & 15,47 & $26,00-44,00$ \\
\hline \multicolumn{5}{|c|}{ Diámetro de tallo $(\mathrm{cm})$} \\
\hline RV1 & $4,95 \mathrm{a}$ & 0,42 & 8,48 & $4,23-5,76$ \\
\hline RV2 & $5,59 \mathrm{~b}$ & 0,52 & 9,30 & $4,61-6,69$ \\
\hline RV3 & $6,17 \mathrm{c}$ & 0,67 & 10,86 & $4,74-7,90$ \\
\hline RV4 & $7,12 \mathrm{~d}$ & 1,05 & 14,75 & $5,96-10,50$ \\
\hline \multicolumn{5}{|c|}{ Volumen de tallo $\left(\mathrm{cm}^{3}\right)$} \\
\hline RV1 & $14,24 \mathrm{a}$ & 2,99 & 21,00 & $9,00-22,00$ \\
\hline RV2 & $20,16 \mathrm{~b}$ & 5,01 & 24,85 & $13,00-34,00$ \\
\hline RV3 & $23,56 \mathrm{~b}$ & 5,28 & 22,41 & $13,00-35,00$ \\
\hline RV4 & $32,80 \mathrm{c}$ & 9,21 & 28,08 & $20,00-58,00$ \\
\hline \multicolumn{5}{|c|}{ Relación tallo/raíz $\left(\mathrm{cm}^{3} / \mathrm{cm}^{3}\right)$} \\
\hline RV1 & $1,26 \mathrm{a}$ & 0,24 & 19,05 & $0,77-1,83$ \\
\hline RV2 & $1,28 \mathrm{a}$ & 0,26 & 20,31 & $0,81-2,00$ \\
\hline RV3 & $1,19 \mathrm{a}$ & 0,26 & 21,85 & $0,65-1,61$ \\
\hline RV4 & $1,23 \mathrm{a}$ & 0,27 & 21,95 & $0,80-1,71$ \\
\hline \multicolumn{5}{|c|}{ Biomasa del tallo (g) } \\
\hline RV1 & $2,27 \mathrm{a}$ & 0,48 & 21,15 & $1,47-3,74$ \\
\hline RV2 & $3,29 \mathrm{~b}$ & 0,78 & 23,71 & $2,10-4,71$ \\
\hline RV3 & $3,88 \mathrm{~b}$ & 1,01 & 26,03 & $1,87-6,50$ \\
\hline RV4 & $5,38 \mathrm{c}$ & 1,75 & 32,53 & $2,92-9,82$ \\
\hline \multicolumn{5}{|c|}{ Biomasa foliar (g) } \\
\hline RV1 & $2,26 \mathrm{a}$ & 0,49 & 21,68 & $1,54-3,42$ \\
\hline RV2 & $2,94 \mathrm{~b}$ & 0,79 & 26,87 & $2,01-5,34$ \\
\hline RV3 & $3,48 \mathrm{~b}$ & 0,65 & 18,68 & $2,49-5,25$ \\
\hline RV4 & $4,74 \mathrm{c}$ & 1,12 & 23,63 & $3,13-7,65$ \\
\hline \multicolumn{5}{|c|}{ Biomasa aérea (g) } \\
\hline RV1 & $4,52 \mathrm{a}$ & 0,92 & 20,35 & $3,05-7,16$ \\
\hline RV2 & $6,22 \mathrm{~b}$ & 1,49 & 23,95 & $4,22-10,05$ \\
\hline RV3 & $7,36 \mathrm{~b}$ & 1,46 & 19,84 & $4,36-10,93$ \\
\hline RV4 & $10,11 \mathrm{c}$ & 2,76 & 27,30 & $6,14-17,47$ \\
\hline \multicolumn{5}{|c|}{ Biomasa radicular $(\mathrm{g})$} \\
\hline RV1 & $2,76 \mathrm{a}$ & 0,51 & 18,48 & $1,95-4,11$ \\
\hline RV2 & $3,70 \mathrm{~b}$ & 0,39 & 10,54 & $2,96-4,43$ \\
\hline RV3 & $4,67 \mathrm{c}$ & 0,61 & 13,06 & $3,71-6,22$ \\
\hline RV4 & $6,41 \mathrm{~d}$ & 1,78 & 27,77 & $4,90-9,78$ \\
\hline
\end{tabular}

* Categorías de volumen radicular para este y los cuadros siguientes: RV1 8-13 cm³; RV2 14-17 cm³; RV3 18-22 cm ; RV4 23-35 $\mathrm{cm}^{3}$ 
BOSQUE 25(2): 17-33, 2004

Efecto del volumen radicular y la tasa de fertilización sobre el comportamiento en terreno...

CUADRO 5

Concentración foliar de nutrientes preplantación por categoría de volumen radicular.

Preplanting nutrient concentration by root-volume category.

\begin{tabular}{|c|c|c|c|c|}
\hline Nutriente & Promedio & Desviación estándar & Coef. de variación (\%) & Rango \\
\hline \multicolumn{5}{|l|}{$\mathrm{N}(\%)$} \\
\hline RV1 & 2,37 & 0,26 & 10,97 & $2,17-2,67$ \\
\hline $\mathrm{RV} 2$ & 2,50 & 0,12 & 4,80 & $2,37-2,60$ \\
\hline RV3 & 2,46 & 0,03 & 1,22 & $2,43-2,48$ \\
\hline RV4 & 2,29 & 0,17 & 7,42 & $2,17-2,49$ \\
\hline \multicolumn{5}{|l|}{$\mathrm{P}(\%)$} \\
\hline RV1 & 0,19 & 0,01 & 5,26 & $0,19-0,20$ \\
\hline RV2 & 0,20 & 0,03 & 15,00 & $0,17-0,22$ \\
\hline $\mathrm{RV} 3$ & 0,21 & 0,02 & 9,52 & $0,20-0,23$ \\
\hline RV4 & 0,22 & 0,01 & 4,55 & $0,22-0,23$ \\
\hline \multicolumn{5}{|l|}{$\mathrm{K}(\%)$} \\
\hline RV1 & 0,63 & 0,06 & 9,52 & $0,60-0,70$ \\
\hline RV2 & 0,70 & 0,20 & 28,57 & $0,50-0,90$ \\
\hline RV3 & 0,73 & 0,06 & 8,22 & $0,70-0,80$ \\
\hline RV4 & 0,77 & 0,06 & 7,79 & $0,70-0,80$ \\
\hline \multicolumn{5}{|l|}{ S (\%) } \\
\hline $\mathrm{RV} 1$ & 0,22 & 0,07 & 31,82 & $0,14-0,27$ \\
\hline RV2 & 0,24 & 0,06 & 25,00 & $0,18-0,28$ \\
\hline RV3 & 0,25 & 0,02 & 8,00 & $0,23-0,26$ \\
\hline RV4 & 0,25 & 0,04 & 16,00 & $0,20-0,28$ \\
\hline \multicolumn{5}{|l|}{$\mathrm{Ca}(\%)$} \\
\hline RV1 & 0,69 & 0,03 & 4,35 & $0,66-0,72$ \\
\hline RV2 & 0,72 & 0,12 & 16,67 & $0,61-0,84$ \\
\hline RV3 & 0,71 & 0,06 & 8,45 & $0,65-0,75$ \\
\hline RV4 & 0,73 & 0,05 & 6,85 & $0,67-0,77$ \\
\hline \multicolumn{5}{|l|}{$\operatorname{Mg}(\%)$} \\
\hline RV1 & 0,19 & 0,02 & 10,53 & $0,17-0,20$ \\
\hline RV2 & 0,19 & 0,04 & 21,05 & $0,15-0,22$ \\
\hline RV3 & 0,17 & 0,02 & 11,76 & $0,16-0,19$ \\
\hline RV4 & 0,18 & 0,01 & 5,56 & $0,17-0,19$ \\
\hline \multicolumn{5}{|l|}{$\mathrm{Na}(\%)$} \\
\hline RV1 & 0,08 & 0,03 & 37,50 & $0,06-0,11$ \\
\hline RV2 & 0,11 & 0,02 & 18,18 & $0,09-0,12$ \\
\hline RV3 & 0,08 & 0,02 & 25,00 & $0,06-0,10$ \\
\hline RV4 & 0,08 & 0,04 & 50,00 & $0,05-0,12$ \\
\hline \multicolumn{5}{|l|}{ B (ppm) } \\
\hline RV1 & 43,30 & 4,42 & 10,21 & $38,20-46,10$ \\
\hline RV2 & 44,80 & 2,23 & $\begin{array}{r}4,98 \\
4,98\end{array}$ & $43,00-47,30$ \\
\hline RV3 & 44,00 & 6,84 & 15,55 & $37,60-51,20$ \\
\hline RV4 & 44,93 & 7,57 & 16,85 & $39,60-53,60$ \\
\hline \multicolumn{5}{|l|}{$\mathrm{Zn}(\mathrm{ppm})$} \\
\hline RV1 & 101,33 & 9,29 & 9,17 & $91,00-109,00$ \\
\hline RV2 & 98,33 & 5,51 & 5,60 & $92,00-102,00$ \\
\hline RV3 & 86,00 & 2,65 & 3,08 & $84,00-89,00$ \\
\hline RV4 & 85,67 & 10,07 & 11,75 & $75,00-95,00$ \\
\hline \multicolumn{5}{|l|}{$\mathrm{Mn}(\mathrm{ppm})$} \\
\hline RV1 & 325,33 & 58,16 & 17,88 & $275,00-389,00$ \\
\hline RV2 & 342,33 & 63,09 & 18,43 & $270,00-386,00$ \\
\hline RV3 & 378,00 & 43,72 & 11,57 & $347,00-428,00$ \\
\hline RV4 & 388,33 & 18,48 & 4,76 & $367,00-399,00$ \\
\hline \multicolumn{5}{|l|}{$\mathrm{Fe}(\mathrm{ppm})$} \\
\hline RV1 & $1.866,67$ & 140,12 & 7,51 & $1.710,00-1.980,00$ \\
\hline RV2 & $1.603,33$ & 86,22 & 5,38 & $1.510,00-1.680,00$ \\
\hline RV3 & $1.470,00$ & 270,74 & 18,42 & $1.160,00-1.660,00$ \\
\hline RV4 & $1.613,33$ & 315,65 & 19,57 & $1.250,00-1.820,00$ \\
\hline \multicolumn{5}{|l|}{$\mathrm{Cu}(\mathrm{ppm})$} \\
\hline RV1 & 19,00 & 5,29 & 27,84 & $15,00-25,00$ \\
\hline RV2 & 14,33 & 2,08 & 14,52 & $12,00-16,00$ \\
\hline RV3 & 13,67 & 2,08 & 15,22 & $12,00-16,00$ \\
\hline RV4 & 35,00 & 33,15 & 94,71 & $12,00-73,00$ \\
\hline
\end{tabular}


BOSQUE 25(2): 17-33, 2004

Efecto del volumen radicular y la tasa de fertilización sobre el comportamiento en terreno...

\section{CUADRO 6}

Contenido foliar de nutrientes preplantación por categoría de volumen radicular. Preplanting nutrient content by root-volume category.

\begin{tabular}{|c|c|c|c|c|}
\hline Nutriente & Promedio & Desviación estándar & Coef. de variación (\%) & Rango \\
\hline \multicolumn{5}{|c|}{$\mathrm{N}$ (mg/100 needles) } \\
\hline RV1 & 6,97 & 0,26 & 3,73 & $6,68-7,16$ \\
\hline RV2 & 7,40 & 0,58 & 7,84 & $7,02-8,06$ \\
\hline RV3 & 7,70 & 0,66 & 8,57 & $7,13-8,43$ \\
\hline RV4 & 7,55 & 0,54 & 7,15 & $6,94-7,97$ \\
\hline \multicolumn{5}{|c|}{ P (mg/100 needles) } \\
\hline RV1 & 0,57 & 0,09 & 15,79 & $0,48-0,66$ \\
\hline RV2 & 0,58 & 0,07 & 12,07 & $0,54-0,66$ \\
\hline RV3 & 0,67 & 0,10 & 14,93 & $0,61-0,78$ \\
\hline RV4 & 0,74 & 0,03 & 4,05 & $0,70-0,77$ \\
\hline \multicolumn{5}{|c|}{$\mathrm{K}$ (mg/100 needles) } \\
\hline RV1 & 1,89 & 0,41 & 21,69 & $1,50-2,31$ \\
\hline RV2 & 2,06 & 0,57 & 27,67 & $1,60-2,70$ \\
\hline RV3 & 2,31 & 0,36 & 15,58 & $2,03-2,72$ \\
\hline RV4 & 2,53 & 0,28 & 11,07 & $2,24-2,80$ \\
\hline \multicolumn{5}{|c|}{$\mathrm{S}$ (mg/100 needles) } \\
\hline RV1 & 0,64 & 0,23 & 35,94 & $0,43-0,89$ \\
\hline RV2 & 0,72 & 0,13 & 18,06 & $0,58-0,84$ \\
\hline RV3 & 0,78 & 0,03 & 3,85 & $0,75-0,81$ \\
\hline RV4 & 0,82 & 0,15 & 18,29 & $0,64-0,91$ \\
\hline \multicolumn{5}{|c|}{$\mathrm{Ca}$ (mg/100 needles) } \\
\hline RV1 & 2,06 & 0,31 & 15,05 & $1,75-2,38$ \\
\hline RV2 & 2,11 & 0,16 & 7,58 & $1,95-2,27$ \\
\hline RV3 & 2,24 & 0,34 & 15,18 & $1,89-2,55$ \\
\hline RV4 & 2,40 & 0,06 & 2,50 & $2,35-2,46$ \\
\hline \multicolumn{5}{|c|}{ Mg (mg/100 needles) } \\
\hline RV1 & 0,55 & 0,07 & 12,73 & $0,50-0,63$ \\
\hline RV2 & 0,55 & 0,06 & 10,91 & $0,48-0,59$ \\
\hline RV3 & 0,54 & 0,07 & 12,96 & $0,46-0,59$ \\
\hline RV4 & 0,59 & 0,02 & 3,39 & $0,58-0,61$ \\
\hline \multicolumn{5}{|c|}{$\mathrm{Na}$ (mg/100 needles) } \\
\hline RV1 & 0,23 & 0,04 & 17,39 & $0,20-0,28$ \\
\hline RV2 & 0,32 & 0,04 & 12,50 & $0,27-0,35$ \\
\hline RV3 & 0,26 & 0,07 & 26,92 & $0,19-0,31$ \\
\hline RV4 & 0,26 & 0,16 & 61,54 & $0,38-0,11$ \\
\hline
\end{tabular}

Continúa 
BOSQUE 25(2): 17-33, 2004

Efecto del volumen radicular y la tasa de fertilización sobre el comportamiento en terreno...

\begin{tabular}{|c|c|c|c|c|}
\hline Nutriente & Promedio & Desviación estándar & Coef. de variación (\%) & Rango \\
\hline \multicolumn{5}{|c|}{ B (mg/100 needles) } \\
\hline RV1 & 0,01 & 0,003 & 30,00 & $0,010-0,015$ \\
\hline RV2 & 0,01 & 0,001 & 10,00 & $0,012-0,014$ \\
\hline RV3 & 0,01 & 0,003 & 30,00 & $0,012-0,017$ \\
\hline RV4 & 0,01 & 0,002 & 20,00 & $0,013-0,017$ \\
\hline \multicolumn{5}{|c|}{$\mathrm{Zn}$ (mg/100 needles) } \\
\hline RV1 & 0,03 & 0,004 & 13,33 & $0,026-0,034$ \\
\hline RV2 & 0,03 & 0,002 & 6,67 & $0,027-0,030$ \\
\hline RV3 & 0,03 & 0,002 & 6,67 & $0,025-0,029$ \\
\hline RV4 & 0,03 & 0,004 & 13,33 & $0,024-0,030$ \\
\hline \multicolumn{5}{|c|}{ Mn (mg/100 needles) } \\
\hline RV1 & 0,10 & 0,03 & 30,00 & $0,08-0,13$ \\
\hline RV2 & 0,10 & 0,01 & 10,00 & $0,09-0,12$ \\
\hline RV3 & 0,12 & 0,02 & 16,67 & $0,10-0,15$ \\
\hline RV4 & 0,13 & 0,01 & 7,69 & $0,12-0,14$ \\
\hline \multicolumn{5}{|c|}{$\mathrm{Fe}(\mathrm{mg} / 100$ needles $)$} \\
\hline RV1 & 0,55 & 0,09 & 16,36 & $0,48-0,65$ \\
\hline RV2 & 0,47 & 0,03 & 6,38 & $0,44-0,50$ \\
\hline RV3 & 0,46 & 0,05 & 10,87 & $0,39-0,49$ \\
\hline RV4 & 0,53 & 0,12 & 22,64 & $0,40-0,62$ \\
\hline \multicolumn{5}{|c|}{$\mathrm{Cu}$ (mg/100 needles) } \\
\hline RV1 & 0,005 & 0,0007 & 14,00 & $0,005-0,006$ \\
\hline $\mathrm{RV} 2$ & 0,004 & 0,0003 & 7,50 & $0,004-0,005$ \\
\hline RV3 & 0,004 & 0,0006 & 15,00 & $0,004-0,005$ \\
\hline RV4 & 0,012 & 0,0119 & 99,17 & $0,004-0,026$ \\
\hline
\end{tabular}

tallo, incremento en altura y diámetro para ningún período de medición. Por lo tanto, para efectos de análisis estadístico el efecto del volumen radicular fue promediado sobre el efecto de la dosis de fertilización y viceversa. La altura inicial adicionó una significativa variación entre las diferentes categorías de volumen radicular y dosis de fertilización para la altura total medida en julio del 2000 ( $\mathrm{p}<0,0001)$, septiembre del 2000 $(\mathrm{p}<0,0001)$ y septiembre del $2001(\mathrm{p}=0,0104)$. El diámetro de tallo inicial agregó una significativa variación entre las diferentes categorías de volumen radicular y dosis de fertilización para el diámetro de tallo medido en julio $(\mathrm{p}<0,0001)$ y septiembre del 2000 ( $p<0,0001)$, pero no para el diámetro de tallo medido en septiembre del 2001 $(\mathrm{p}=0,0953)$.
Efecto del volumen radicular: El volumen radicular afectó significativamente la supervivencia de las plantas transcurridas dos temporadas de plantadas en terreno $(\mathrm{p}=0,0094)$ y no afectó a esta variable al término de la primera temporada de crecimiento. Al término de la segunda temporada de crecimiento, las plantas en la categoría RV4 presentaron las más altas tasas de supervivencias mientras que aquellas en RV3, RV2 y RV1 las menores (cuadro 7).

Al igual que para la morfología inicial de las plantas, la altura y el diámetro inicial de las plantas medidas en terreno varió significativamente $(p<0,0001)$ entre categorías de volumen radicular. Inicialmente las plantas en RV4 eran significativamente más altas y más gruesas que las plantas en RV3, RV2 y RV1, las que también difirieron significativamente entre ellas para ambas va- 
BOSQUE 25(2): 17-33, 2004

Efecto del volumen radicular y la tasa de fertilización sobre el comportamiento en terreno...

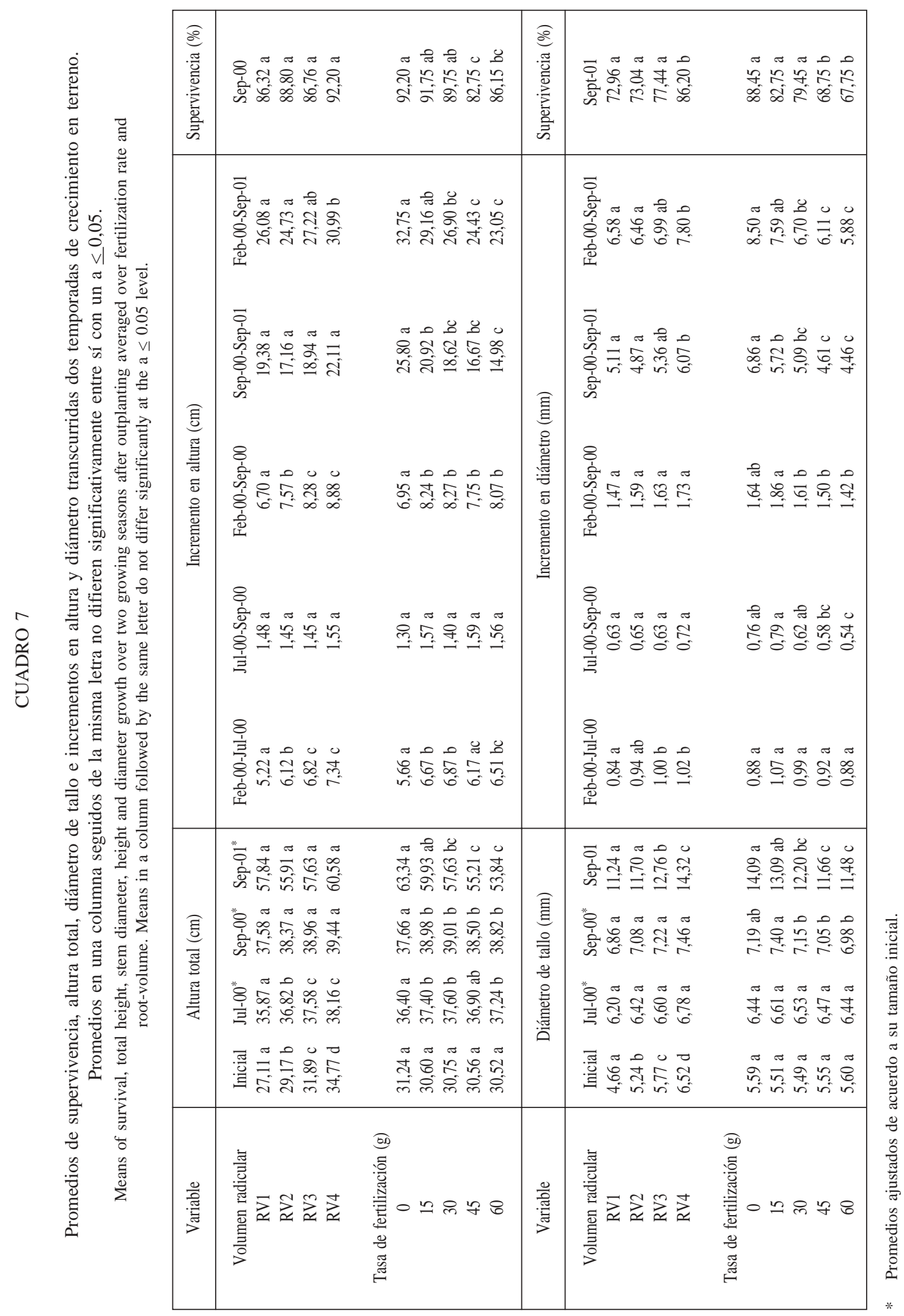


BOSQUE 25(2): 17-33, 2004

Efecto del volumen radicular y la tasa de fertilización sobre el comportamiento en terreno...

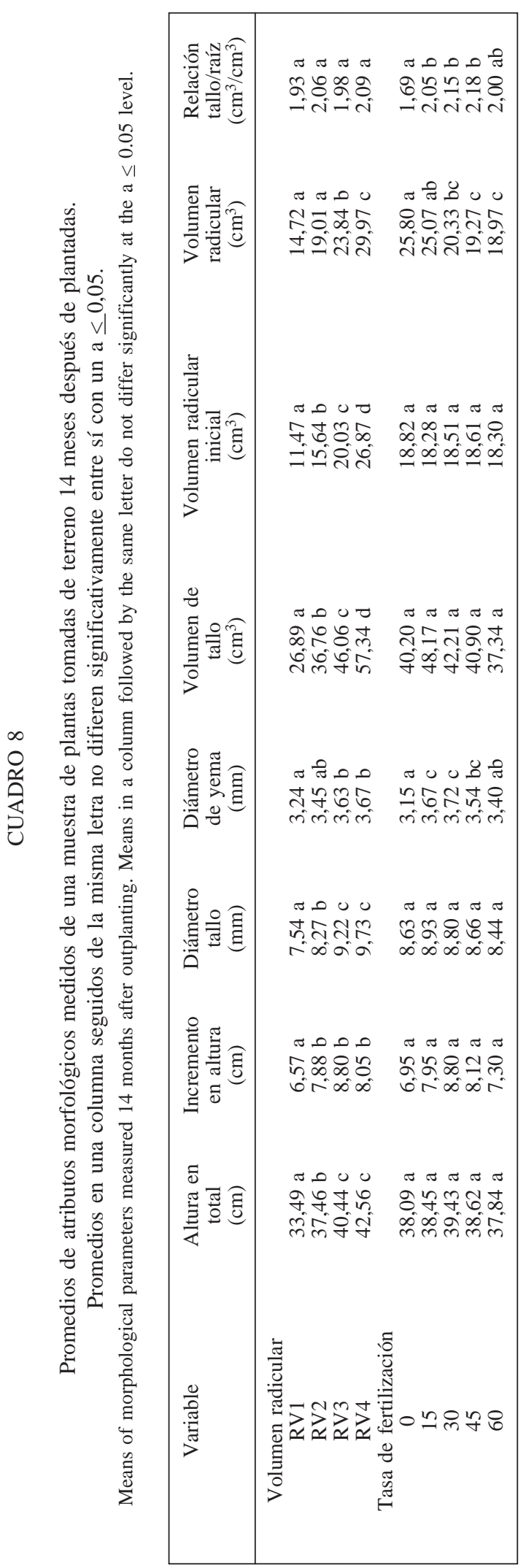

riables. Al término de la primera y segunda temporadas de crecimiento, las plantas en RV4 presentaban la mayor altura y el mayor diámetro; sin embargo, las diferencias entre medias eran estadísticamente significativas sólo para el diámetro. De igual forma, las plantas en RV4 tuvieron los mayores incrementos en altura y diámetro entre todos los períodos de crecimiento, resultando en los mayores crecimientos acumulados transcurridas dos temporadas de crecimiento en terreno (cuadro 7).

Los resultados obtenidos de la muestra de plantas tomadas en terreno después de 14 meses de plantadas coinciden con los datos obtenidos del experimento completo. Las plantas en RV4 presentaron los mejores atributos morfológicos (cuadro 8).

Efectos de la fertilización: La supervivencia de las plantas difirió significativamente entre las dosis de fertilización al término de la primera $(\mathrm{p}=0,0077)$ y segunda $(\mathrm{p}<0,0001)$ temporadas de crecimiento. Al término de ambas temporadas, las plantas no fertilizadas presentaron la mayor supervivencia, pero no difirieron significativamente de aquellas fertilizadas con menos de $30 \mathrm{~g}$ por planta (cuadro 7).

El crecimiento en altura fue estimulado por la fertilización durante el primer año. Aquellas plantas no fertilizadas crecieron significativamente menos en altura que las fertilizadas. Durante la segunda temporada de crecimiento, el aumento de la dosis de fertilización redujo significativamente el incremento en altura de las plantas, resultando en una reducción significativa del crecimiento en altura de las plantas transcurridas dos temporadas desde plantadas (cuadro 7).

En el transcurso del primer año, el crecimiento diamétrico fue estimulado mayormente con la aplicación de $15 \mathrm{~g}$ de fertilizante por planta. Al igual que con la altura, el aumento de la dosis de fertilización redujo significativamente el crecimiento en diámetro durante la segunda temporada de crecimiento en terreno, resultando con el menor crecimiento acumulado (cuadro 7).

Los resultados del muestreo de plantas desde terreno 14 meses desde plantadas (marzo 2001) mostraron que a esa fecha la fertilización no afectó la longitud del tallo ( $\mathrm{p}=0,7361)$, crecimiento en altura $(\mathrm{p}=0,0515)$, diámetro $(\mathrm{p}=0,5087)$ y volumen de tallo $(\mathrm{p}=0,1123)$. El aumento de la dosis de fertilización redujo significativamente el volumen 
BOSQUE 25(2): 17-33, 2004

Efecto del volumen radicular y la tasa de fertilización sobre el comportamiento en terreno...

radicular $(\mathrm{p}=0,0164)$, afectando con ello la relación tallo-raíz de las plantas ( $\mathrm{p}=0,0002)$. La relación tallo-raíz fue mayor en aquellas plantas fertilizadas con $45 \mathrm{~g}$ de fertilizante y menor en las que no fueron fertilizadas. El diámetro de la yema terminal también fue afectado por la fertilización $(p=0,0002)$. Fue mayor en aquellas plantas fertilizadas con $30 \mathrm{~g}$ y menor en las plantas sin fertilización (cuadro 8).

\section{DISCUSION}

Efecto del volumen radicular: Los resultados de este estudio fueron consistentes con los resultados obtenidos por otros investigadores $(8,9,13)$, en relación a la positiva correlación existente entre el volumen radicular y otros atributos morfológicos, tales como el diámetro y volumen del tallo y la biomasa de algunos componentes de las plantas (cuadro 3). Sin embargo, tal como lo mencionó Rose et al. (10), a pesar de un alto coeficiente de correlación entre el volumen radicular y el diámetro del tallo $(\mathrm{r}=0,81)$, el diámetro del tallo por sí solo es un criterio insuficiente para estimar calidad de plantas, ya que algunas plantas con grandes diámetros pueden tener pequeños sistemas radiculares y viceversa, producto de pérdidas de masa radicular durante el proceso de extracción y clasificación. Si la clasificación de plantas sólo se basa en diámetro, plantas con grandes diámetros y pobres sistemas radiculares podrían ser enviadas a sitios de plantación.

La alta tasa de supervivencia al término de la primera temporada de crecimiento puede ser atribuida a la cuidadosa manipulación y plantación al momento de la instalación del estudio. La precipitación durante el año 2001 fue considerablemente menor que la del año 2000 (cuadro 3), lo que puede haber influido en la reducción de las tasas de supervivencia al término de la segunda temporada de crecimiento (cuadro 7).

Al igual que los resultados de estudios anteriores $(8,9,10)$, este estudio demostró que el volumen radicular es un buen predictor de comportamiento de las plantas en terreno. Aquellas plantas con mayor volumen radicular al momento de la plantación presentaron mayores tasas de supervivencia y crecimiento inicial en altura y diámetro que aquellas con menor volumen radicular, transcurridas la primera y segunda temporadas de creci- miento (cuadros 7 y 8). Esto se explica por el hecho de que inmediatamente después de establecida la planta en terreno, las plantas dependen de las características de sus raíces para la absorción de agua y nutrientes del suelo. Las plantas con mayor volumen radicular son capaces de tolerar mejor el shock de trasplante (13) debido a una mayor conductividad hidráulica de las raíces (7). En la medida que la temporada de crecimiento avanza la temperatura del suelo aumenta; plantas bien plantadas de mayor volumen radicular debieron haber comenzado la absorción de agua del suelo antes que aquellas con menor volumen radicular (7). Ante una mayor oferta de agua, aquellas plantas con mayor volumen radicular absorbieron y distribuyeron más nutrientes hacia polos de crecimiento que aquellas con menor volumen radicular (14).

Efectos de la fertilización: A pesar de que las plantas fertilizadas crecieron significativamente más en altura que las no fertilizadas durante la primera temporada de crecimiento (cuadro 7), la magnitud de las diferencias no fue biológicamente importante. La escasa respuesta a la fertilización durante el primer año pudo haberse debido a baja temperatura y humedad disponible en el suelo, lo que dificultó el correcto funcionamiento de los mecanismos de entrega del fertilizante aplicado.

El mecanismo de entrega de nutrientes de los fertilizantes de entrega controlada con cubierta sintética es dependiente de la temperatura. Los períodos de entrega de nutrientes especificados por el fabricante del fertilizante son estimados a una temperatura de $21^{\circ} \mathrm{C}$. Aparte del elevado valor de temperatura registrado en abril del 2000, probablemente porque el sensor estuvo en la superficie del suelo por algunos días, las temperaturas máximas del suelo en el sitio experimental sólo alcanzaron en promedio los $21^{\circ} \mathrm{C}$ durante el mes de agosto del 2000 (cuadro 3). A esa fecha, se estimó a partir de un estudio en el que se midió el efecto residual del mismo fertilizante, establecido en el mismo sitio, que sólo el 30\% del fertilizante aplicado había sido entregado a la planta (figura 2). Aproximadamente sólo un $20 \%$ del fertilizante habría sido entregado a las plantas hasta junio, época del año en que la humedad aún no limita el crecimiento de pino oregón en esta zona de su distribución. Sin embargo, la escasa proporción de nutrientes entregados al comienzo de la temporada de crecimiento fue suficiente para establecer diferencias signifi- 
BOSQUE 25(2): 17-33, 2004

Efecto del volumen radicular y la tasa de fertilización sobre el comportamiento en terreno...

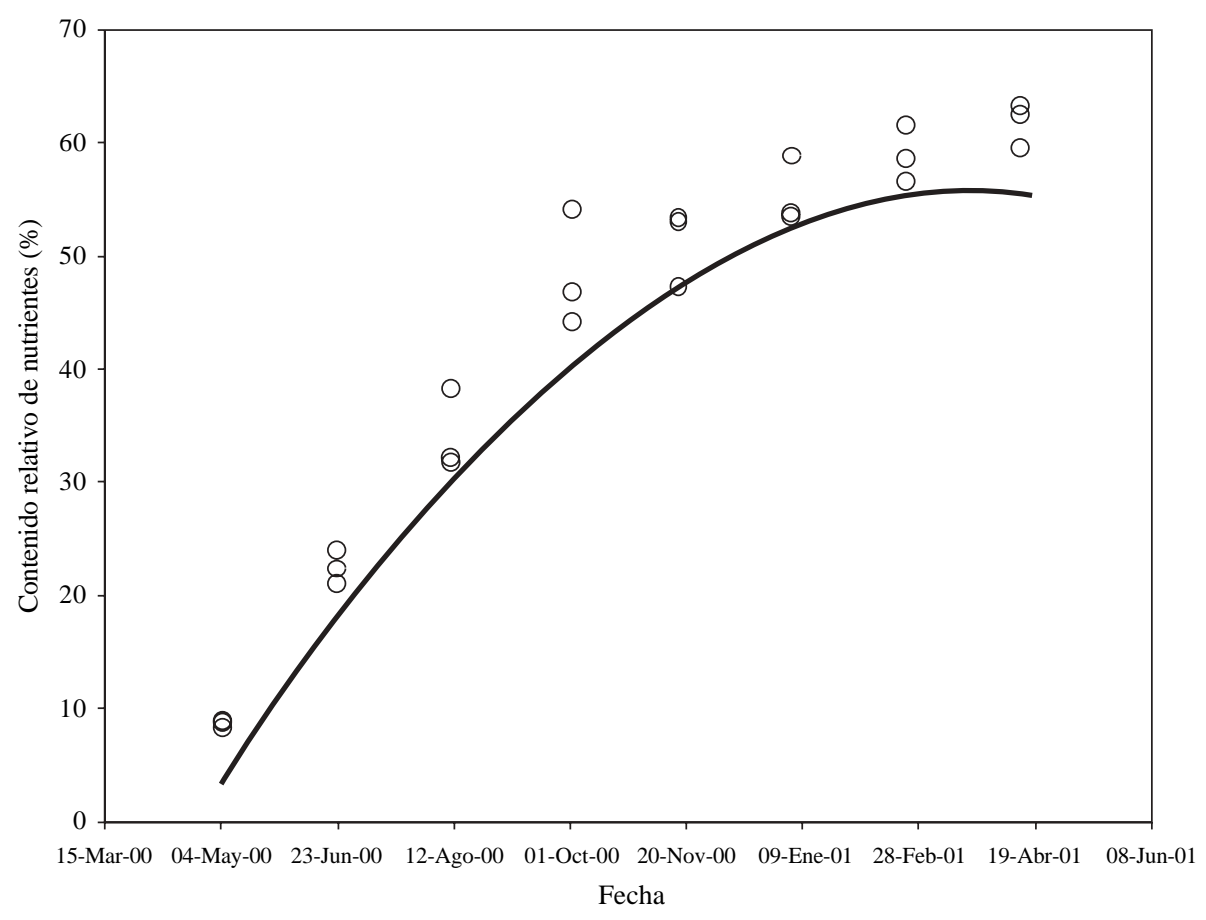

Figura 2. Proporción del contenido inicial de nutrientes (base peso seco) liberados por el fertilizante aplicado en este estudio.

Relative fertilizer release content by weight across time for the fertilizer used at the study.

cativas en la altura total de las plantas en relación a las no fertilizadas.

Otra posible explicación de la baja respuesta a la fertilización durante el primer año de crecimiento puede atribuirse al shock de trasplante producto de la escasa disponibilidad de agua. Al término de la primera temporada de crecimiento la mayoría de las plantas exhibió signos de shock de trasplante, atrofiamiento del crecimiento apical, abundante número de acículas por unidad de longitud de tallo y reducción de su tamaño (13). Las mediciones de incremento en altura (cuadro 8) son similares a aquellas reportadas por Haase y Rose (13) al cultivar plantas de pino oregón entre 6 y $18 \%$ de humedad del suelo. Ante tales condiciones de escasez de humedad, la absorción de nutrientes pudo haber sido considerablemente reducida (14). Van den Driessche (18), al comparar el efecto de diferentes fertilizantes de entrega controlada aplicados al momento de la plantación, reporta una reducción en el contenido foliar de nutrientes de plantas a raíz desnuda de pino oregón seis meses después de plantadas. Al término de la primera temporada de crecimiento, las plantas con sus tratamientos de fertilización presentaban concentraciones foliares marginalmente superiores a las no fertilizadas con pequeñas diferencias en crecimiento. De igual manera, Walker $(19,20)$ reportó pobres respuestas en crecimiento a la fertilización al término de la primera temporada de crecimiento en plantas de pino jeffrey con la aplicación de varios fertilizantes de entrega controlada aplicados al momento de la plantación en un sitio seco en el Este de la Sierra Nevada.

Al comienzo de la segunda temporada de crecimiento, el aumento de la dosis de fertilización disminuyó significativamente el volumen radicular de las plantas (figura 3), produciendo un aumento significativo de la relación tallo-raíz (figura 4). Debido a la dependencia de la temperatura de los fertilizantes de entrega controlada para la liberación de nutrientes, el fertilizante continuó liberando nutrientes durante el verano, otoño e invierno de 2001, aumentando con ello la concentración de sales a nivel de la rizósfera (figura 2). La baja cantidad de lluvias durante el invierno de 2001, comparada con el invierno anterior, y la escasa precipitación durante el verano de 2000 (cuadro 3) 
BOSQUE 25(2): 17-33, 2004

Efecto del volumen radicular y la tasa de fertilización sobre el comportamiento en terreno...

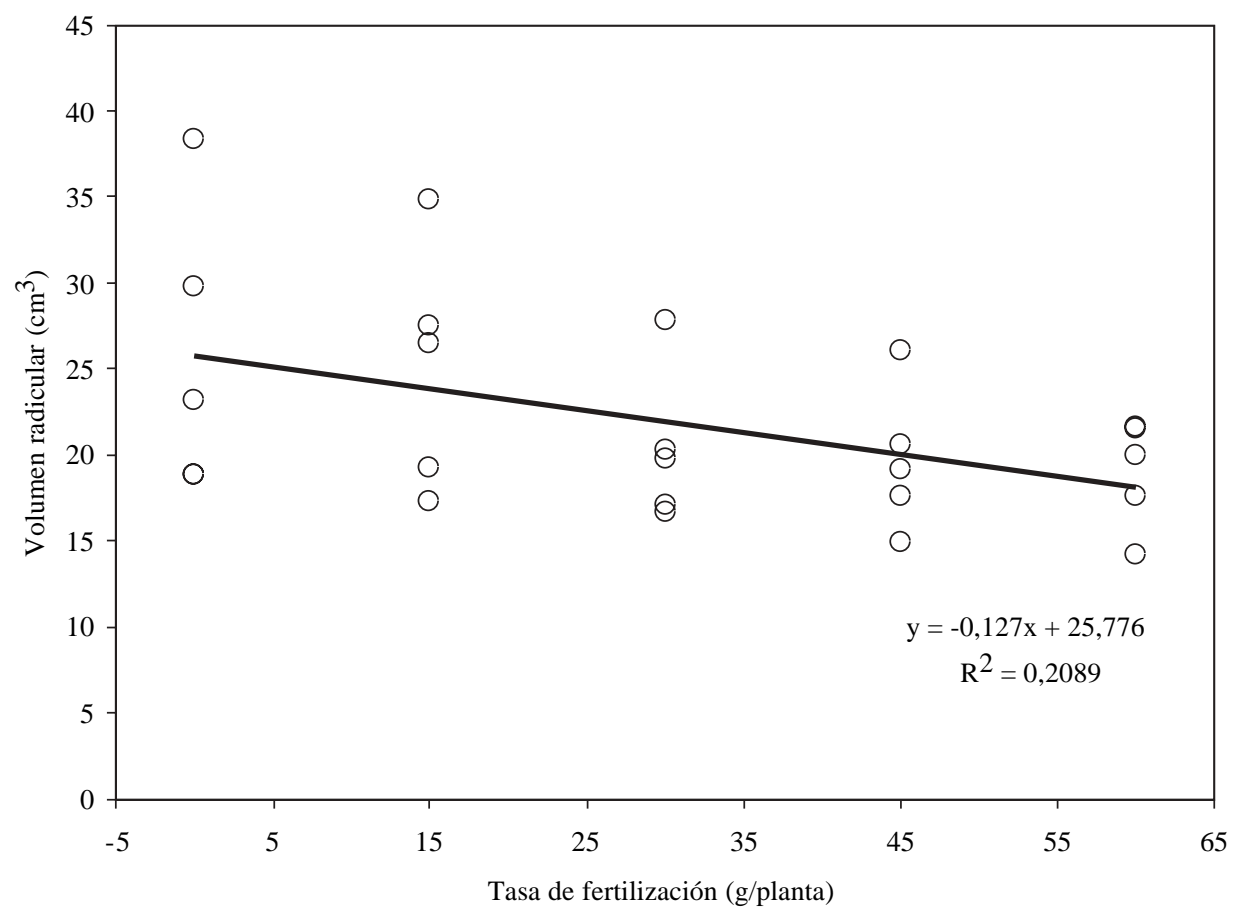

Figura 3. Efecto de la tasa de fertilización sobre el volumen radicular de las plantas transcurrida la primera temporada de crecimiento en terreno.

Effect of fertilization rate on seedling root volume after one growing season in the field.

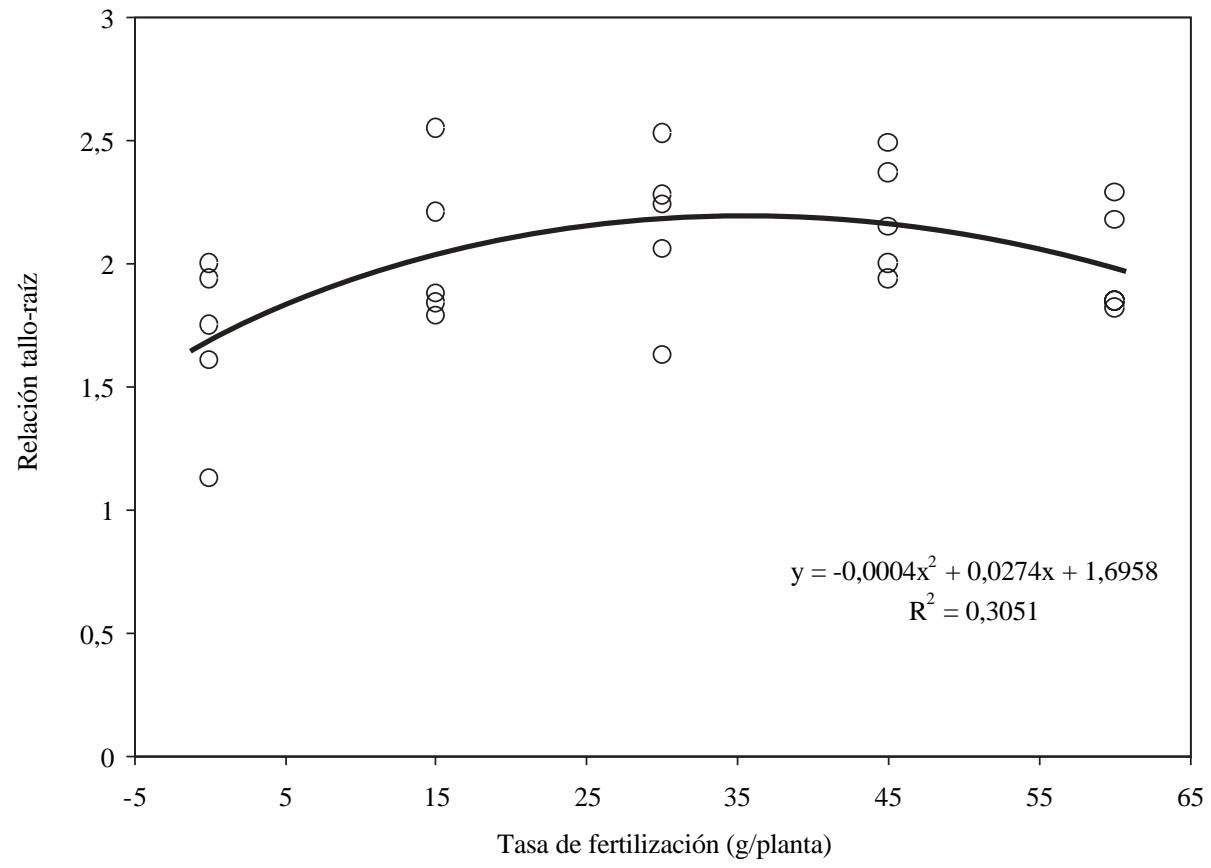

Figura 4. Efecto de la tasa de fertilización sobre la relación tallo-raíz de las plantas transcurrida la primera temporada de crecimiento en terreno.

Effect of fertilization rate on seedling shoot-root ratio after one growing season in the field. 
también debieron haber contribuido al aumento de la salinidad en la rizósfera.

Al comenzar la segunda temporada de crecimiento (abril 2001), más del 50\% (basado en el peso) del fertilizante aplicado había sido entregado (figura 2). Nitrógeno ( $80 \%$ de su contenido inicial), potasio (60\% de su contenido inicial) y azufre $(30 \%$ de su contenido inicial) fueron los principales nutrientes liberados. Menos del 20\% del contenido de fósforo y magnesio habría sido liberado (figura 5). La aplicación de dosis crecientes de fertilizantes nitrogenados bajo las raíces de las plantas debió haber restringido el crecimiento radicular, reduciendo su volumen y aumentando la relación tallo-raíz. Olsthoorn et al. (21) reportaron que aplicaciones de $340 \mathrm{~kg}$ por hectárea de sulfato de amonio a plantas de pino oregón redujeron la longitud y longitud específica (relación de longitud de raíz-peso seco raíz) de las raíces, especialmente en zonas de alta acumulación de amonio. Agregan, además, que el crecimiento del tallo fue estimulado en una magnitud tal, que la relación tallo-raíz de las plantas fertilizadas a la tasa más alta casi duplicó a las no fertilizadas. De Visser y
Keltjens $(22,23)$, trabajando con la misma especie obtuvieron resultados similares.

Todos los autores coinciden en señalar que la causa de la alteración en el desarrollo radicular de las plantas producida por fertilizantes amónicos se debe a un aumento de la concentración de aluminio e hidrógeno, producto de la acidificación del suelo durante la nitrificación del amonio y la emisión de hidrógeno por la planta mientras absorbe amonio. Desgraciadamente, en este estudio, sólo las características químicas del suelo antes de la plantación fueron determinadas, lo que impidió determinar cambios en el suelo después de la fertilización.

Jacobs (24) encontró también, para pino oregón, que el peso seco de las raíces laterales y el número de raíces activas sobre una capa de Osmocote (159-12) con efecto residual de 5-6 meses fueron reducidas significativamente al incrementar las dosis de fertilización, reduciendo la biomasa y el volumen radicular de las plantas. Este autor atribuyó sus resultados a un incremento localizado de la concentración de sales que pudo haber dañado los meristemas apicales de las raíces producto de una reducción del potencial osmótico.

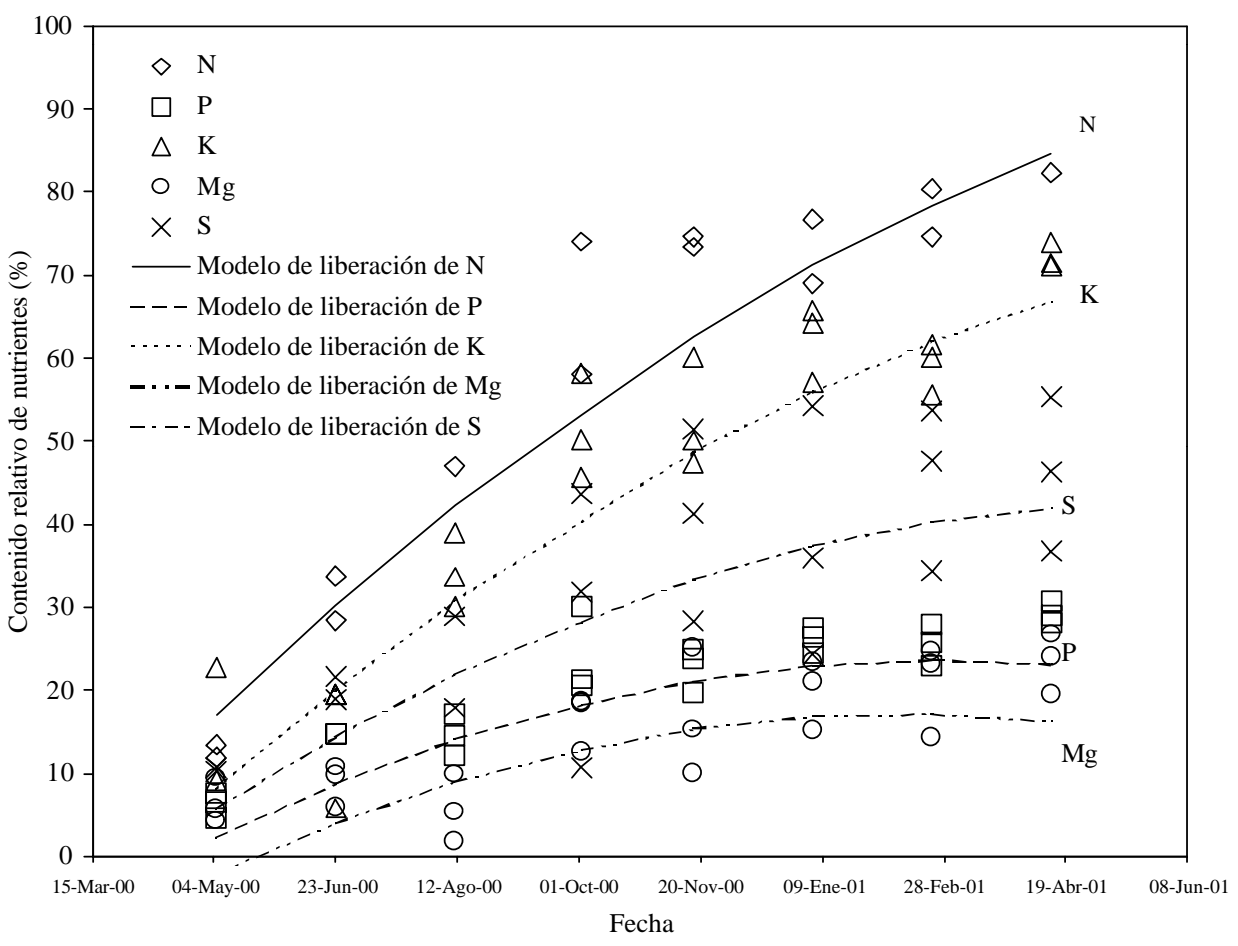

Figura 5. Liberación de macronutrientes a través del tiempo del fertilizante aplicado en el estudio. Relative macronutrient release across time for the fertilizer used in the study. 
BOSQUE 25(2): 17-33, 2004

Efecto del volumen radicular y la tasa de fertilización sobre el comportamiento en terreno...

Independiente de la causa de la reducción del crecimiento radicular con el aumento de la fertilización, las plantas fertilizadas con la más alta dosis presentaron las más altas relaciones tallo-raíz. En la medida que la temporada de crecimiento avanzaba y la disponibilidad de agua disminuía, aquellas plantas con altas relaciones tallo-raíz no fueron capaces de reponer las pérdidas de agua por transpiración. Jacobs (24) analizó las relaciones hídricas en una muestra de estas plantas y midió valores más altos de potencial hídrico y menor conductividad estomática durante el verano del 2000 en las plantas que fueron fertilizadas a la mayor tasa que en aquellas no fertilizadas.

En este estudio, ya que tanto el crecimiento en altura como el volumen aéreo no fue afectado por la fertilización al comienzo de la segunda temporada de crecimiento (cuadro 8), aplicaciones en dosis crecientes de fertilización a la rizósfera de plantas de pino oregón pudieron haber dañado sus sistemas radiculares, reduciendo su capacidad para absorber agua y nutrientes desde el suelo.

\section{CONCLUSIONES}

Los resultados de este estudio confirmaron que el volumen radicular es un buen predictor del comportamiento de las plantas en terreno. Aquellas plantas con el mayor volumen radicular RV4, al momento de la plantación, presentaron las más altas tasas de supervivencia y crecimiento en altura y diámetro transcurridas dos temporadas de crecimiento en terreno. Viveristas debieran poner particular énfasis en implementar prácticas de manejo tendientes a maximizar el desarrollo radicular de las plantas durante el cultivo y reducir las pérdidas de masa radicular durante la extracción y manipulación de las plantas. De igual manera los supervisores de plantaciones debieran capacitar a los plantadores a no podar los sistemas radiculares abundantes al momento de la plantación, ya que, como lo demostró este estudio, cada unidad de volumen radicular cuenta en el establecimiento de plantaciones exitosas. Es igualmente importante producir plantas balanceadas en su relación tallo-raíz. Durante la segunda temporada de crecimiento, en este estudio, aquellas plantas con una alta relación tallo-raíz, producida por una nutrición desbalanceada, afectó negativamente su crecimiento.
Debido a bajas temperaturas de suelo, las que limitaron la liberación de nutrientes desde los fertilizantes de entrega controlada, la fertilización con fertilizantes de entrega controlada promovió sólo levemente el crecimiento de las plantas durante la primera temporada de crecimiento. Durante la segunda temporada de crecimiento, el aumento en las dosis de fertilización disminuyó las tasas de supervivencia y crecimiento de las plantas, debido a una reducción en la resistencia al estrés hídrico y una restricción del desarrollo radicular de las plantas producto de un exceso de sales en la rizósfera. Por lo tanto, basado en los resultados de este estudio, obtenidos al cabo de dos temporadas de crecimiento en terreno, sería recomendable no aplicar este tipo de productos a la zona radicular de pino oregón establecidos en sitios en los que estarán expuestos a condiciones de sequía moderada a severa.

\section{BIBLIOGRAFIA}

(1) ROTH, B., M. NEWTON. Survival and growth of Douglasfir relating to weeding, fertilization, and seed source. Western Journal of Applied Forestry. 1996, vol. 11, p. 62-69.

(2) JOHNSON, J., M. CLINE, M. Seedling quality of southern pines. In: DURYEA, M., DOUGHERTY, P., eds. Forest Regeneration Manual. Dordrecht, the Netherlands: Kluwer Academic Publishers. 1991. p. 143-159.

(3) RITCHIE, G. Assessing seedling quality. In: DURYEA, M., LANDIS, T., eds. Forest Nursery Manual: Production of Bareroot Seedlings. The Hague: Martinus Nijhoff/Dr. W. Junk Publishers. 1984. p. 243-259.

(4) MEXAL, J., T. LANDIS. Target seedling concepts: Height and diameter. In: ROSE R., CAMPBELL, S., LANDIS, T., eds. Proceedings, Target Seedling Symposium, Combined Meeting of the Western Forest Nursery Associations; 1990 August 15-17; Roseburg, OR. GTR-RM-200. Ft. Collins, CO: USDA Forest Service, Rocky Mountain Forest and Range Experiment Station. 1990. p. 17-36.

(5) MEXAL, J., D. SOUTH. Bareroot seedling culture. In: DURYEA, M., DOUGHERTY, P., eds. Forest Regeneration Manual. Dordrecht, the Netherlands: Kluwer Academic Publishers. 1990. p. 89-115.

(6) BOYER, J., D. SOUTH. Excessive seedling height, high shoot-to-root ratio, and benomyl root dip reduce survival of stored loblolly pine seedlings. Tree Planters' Note. 1987. $\mathrm{N}^{\circ} 38$, p. $19-22$

(7) CARLSON, W. Root system considerations in the quality of loblolly pine seedlings. Southern Journal of Applied Forestry. 1986. № 10, p. 87-92.

(8) ROSE, R., M. ATKINSON, J. GLEASON, T. SABIN. Root volume as a grading criterion to improve field performance of Douglas-fir seedlings. New Forests. 1991. $\mathrm{N}^{\circ} 5$, p. $195-209$.

(9) ROSE, R., J. GLEASON, M. ATKINSON, T. SABIN Grading ponderosa pine seedlings for outplanting according to their root volume. Western Journal of Applied Forestry. 1991. № 6, p. 11-15. 
(10) ROSE, R., D. HAASE, F. KROIHER, T. SABIN. Root volume and growth of ponderosa pine and Douglas-fir seedlings: a summary of eight growing seasons. Western Journal of Applied Forestry. 1997. № 12, p. 69-73.

(11) HARRINGTON, J., J. MEXAL, J. FISHER. Volume displacement provides a quick and accurate way to quantify new root production. Tree Planters' Notes. 1994. N $\mathrm{N}^{\circ}$, p. 121-124.

(12) RACEY, G. A comparison of planting stock characterization with root area index, volume and dry weight. Forestry Chronicl. 1985. № 61, p. 64-70.

(13) HAASE, D., R. ROSE, R. Soil moisture stress induces transplant shock in stored and unstored 2+0 Douglas-fir seedlings of varying root volumes. Forest Science. 1993. $\mathrm{N}^{\circ} 2$, p. $275-294$

(14) HAASE, D., R. ROSE. 1994. Effect of soil water content and initial root volume on the nutrient status of $2+0$ Douglasfir seedlings. New Forests. 1993, vol. 8, p. 265-277.

(15) CARLSON, W. Effects of controlled-release fertilizers on the shoot and root development of outplanted western hemlock seedlings. Canadian Journal of Forest Research. 1981, vol. 11, p. 752-757.

(16) CARLSON, W., C. PREISIG. Effects of controlled-release fertilizers on the shoot and root development of Douglasfir seedlings. Canadian Journal of Forest Research. 1981, vol. 11, p. 230-242.

(17) JOKELA, E., L. ALLEN, W. MCFEE. Fertilization of southern pines at establishment. In: DURYEA, M., DOUGHERTY, P., eds. Forest Regeneration Manual. Dordrecht, the Netherlands: Kluwer Academic Publishers. 1991. p. 263-277.
(18) VAN DEN DRIESSCHE, R. Response of Douglas-fir [Pseudotsuga menziesii (Mirb.) Franco] to some different fertilizers applied at planting. New Forests. 1988, vol. 2, p. 89-110.

(19) WALKER, R. Artificial regeneration of Jeffrey pine in the Sierra Nevada: growth, nutrition and water relations as influenced by controlled release fertilization and solar protection. Journal of Sustainable Forestry. 1999, vol. 9, p. 23-38.

(20) WALKER, R. Reforestation of an Eastern Sierra Nevada surface mine with containerized Jeffrey pine: seedling growth and nutritional responses to controlled release fertilization and ectomycorrhizal inoculation. Journal of Sustainable Forestry. 1999, vol. 9, p. 127-147.

(21) OLSTHOORN, A., W. KELTJENS, B. VAN BAREN, M. HOPMAN. Influence of ammonium on fine root development and rhizosphere $\mathrm{pH}$ of Douglas-fir seedlings in sand. Plant and Soil. 1991, vol. 133, p. 75-81.

(22) DE VISSER, P., W. KELTJENS. Growth and nutrient uptake of Douglas-fir seedlings at different rates of ammonium supply, with or without additional nitrate and other nutrients. Netherlands Journal of Agricultural Science. 1993, vol. 41, p. 327-341.

(23) DE VISSER, P., W. KELTJENS, G. FINDENEGG. Transpiration and drought resistance of Douglas-fir seedlings exposed to excess ammonium. Trees. 1996, vol. 10, p. 301-307.

(24) JACOBS, D. Influence of root architectural development on Douglas-fir seedling morphology and physiology. Ph.D. thesis, Oregon State University, Corvallis, OR. 2002. p. $120-153$. 